\title{
49. Laminin in Animal Models for Muscular Dystrophy Defect of Laminin $M$ in Skeletal and Cardiac Muscles and Peripheral Nerve of the Homozygous Dystrophic dy/dy Mice
}

\author{
By Kiichi ARAhata, ${ }^{* 1,+)}$ Yukiko K. HaYAShI, ${ }^{* 1, * 2)}$ Ritsuko KoGA, ${ }^{* 1)}$ \\ Kanako GoTo, ${ }^{* 1)}$ Je Hyeon LeE, ${ }^{* 1)}$ Yuko MiYagoe, ${ }^{* 1)}$ Hiroko ISHII, ${ }^{* 3)}$ \\ Toshifumi Tsukahara, ${ }^{* 1)}$ Shin'ichi Takeda, ${ }^{* 1)}$ Man Woo, ${ }^{* 4)}$ \\ Ikuya NonAKA, ${ }^{* 1)}$ Tetsuya MATSUZAKI, ${ }^{* 1)}$ \\ and Hideo SUGITA ${ }^{* 1)}$
}

(Communicated by Setsuro Ebashi, M. J. A., Dec. 13, 1993)

\begin{abstract}
We have immunocytochemically shown a significant reduction in the amount of laminin M (or merosin; a tissue-restricted basal lamina protein expressed in striated muscle, Schwann cells, and placental trophoblast) in the skeletal muscle of Fukuyama type congenital muscular dystrophy (FCMD). ${ }^{1)}$ To inquire into the role of laminin $M$ in the process of muscular dystrophies, we examined laminin $M$ in several animal models that cause muscular dystrophy. Immunofluorescent, immunoblotting, and electron microscopic analyses have revealed that laminin $\mathrm{M}$ is missing from skeletal and cardiac muscles and peripheral nerve in the affected homozygous C57BL/6J-dy/dy mice, but not in the non-affected heterozygous $D y / d y$ and the other dystrophic animal models including $m d x$ mice, BIO 14.6 hamsters, and line 413 chickens. In the $d y / d y$ mice, laminin M mRNA is not detected by Northern blotting, but becomes detectable by RT-PCR amplification. Other components of the basal lamina such as laminin B, beta-integrin, type IV collagen, and fibronectin are normally expressed in all animals examined, including the $d y / d y$ mice. These observations strongly suggest that laminin $M$ defect is primarily responsible for the pathogenesis of muscle fiber damage and dysmyelination of the dystrophic $d y / d y$ mice.
\end{abstract}

Key words: Muscular dystrophy; Basal lamina; Laminin M; Dystrophic $d y$ mouse; $m d x$ mouse; Dystrophic hamster; Dystrophic chicken; Animal model.

Introduction. In 1955, a progressive fatal murine muscular dystrophy had been discovered as a spontaneous mutant of the strain 129 mice at the Jackson Laboratories, and designated as dystrophia muscularis $(d y) .{ }^{2}$ The disease is inherited in an autosomal recessive fashion and a gene responsible for $d y$ has been assigned to mouse chromosome 10 where the gene for utrophin is located. ${ }^{3)}$ But utrophin is present in the muscle membrane of the $d y / d y$ mice $^{4), 5)}$ Dystrophin and dystrophin-associated glycoproteins (DAGs) have well been studied in muscular dystrophy models, e.g., the mdx mice, ${ }^{6)-9)}$ BIO 14.6 hamsters,${ }^{10), 11)}$ line 413 chickens, ${ }^{9,12)}$ and $d y / d y$ mice, ${ }^{4), 5)}$ but other gene products in these animals have to be further investigated.

In the $d y$ mice, three genotypes occur, i.e., $d y / d y$ (affected homozygous), $D y / D y$

*1) National Institute of Neuroscience, NCNP, Tokyo 187, Japan.

*2) Department of Neurology, Juntendo University Hospitals, Tokyo 113, Japan.

*3) Tokyo Metropolitan Higashiyamato Medical Center for the Handicapped, Tokyo, Japan.

*4) Department of Pediatrics, Kansai Medical College, Kyoto, Japan.

†) Correspondence to: K. Arahata. Department of Neuromuscular Research, National Institute of Neuroscience, NCNP, 4-1-1 Ogawa-higashi, Kodaira, Tokyo 187, Japan. 
(unaffected homozygous) and $D y / d y$ (unaffected heterozygous carrier). ${ }^{13)}$ Homozygous $d y / d y$ mice show morphological similarities to the severe human muscular dystrophies such as Duchenne (DMD) and Fukuyama (FCMD) types, except dysmyelination in the proximal part of sciatic nerves and the ventral and dorsal spinal roots. ${ }^{14-18)}$

Recently, we found a marked reduction in the amount of laminin $\mathrm{M}$ in skeletal muscle of the patients with $\mathrm{FCMD}^{1)}$ in sharp contrast to the preservation of other proteins in the basal lamina by immunofluorescence, implying a primary or central role of laminin $\mathrm{M}$ in the early stage muscle fiber damage of FCMD patients.

In the present study, we have examined the changes of laminin $\mathrm{M}$ and other protein components of the basal lamina and the membrane-associated cytoskeletal protein, dystrophin, in the $\mathrm{C} 57 \mathrm{BL} / 6 \mathrm{~J}-d y / d y$ mice and other animal models of muscular dystrophy.

Materials and methods. Fertilized ova (2-cell ovum) of the dystrophic homozygous $d y / d y$ mice (C57BL/6J-dy/dy) were purchased from Central Institute for Experimental Animals (Kawasaki, Japan). The inseminated ova were then transferred to the salpinx of the non-dystrophic female ICR mice, and litters of dystrophic $d y / d y, D y / d y$, and its age-matched control C57BL/6J mice were obtained. Mice were sacrificed by cervical dislocation. Muscles and other tissues were examined up to 3 months after birth. Muscles from dystrophic chickens (line 413), hamsters (BIO 14.6), and mice ( $m d x$ ) were studied together.

Antibodies. Anti-laminin M antibodies used in this study are polyclonal affinitypurified rabbit antibody against the C-terminal segment of human laminin $M^{1,{ }^{1,19)}}$ and polyclonal rabbit antibody against the N-terminal segment of mouse laminin $M{ }^{19}{ }^{19}$ (kindly presented by Dr. Engvall). Polyclonal rabbit anti-mouse laminin B (Upstate Biotech. Inc., U.S.A.), and antibodies against type IV collagen (Advance Inc., Tokyo), beta-integrin (Chemicon Inc., U.S.A.), fibronectin (Chemicon Inc., U.S.A.), and dystrophin 6-10 ${ }^{1)}$ were also used simultaneously.

Affinity-purified fluorescein isothiocyanate (FITC)-labeled goat $\mathrm{F}\left(\mathrm{ab}^{\prime}\right)^{2}$ anti-rabbit IgG was obtained from Tago Inc., and used as the second layer antibody.

Immunocytochemistry. Immunocytochemical analysis of each tissue was accomplished as previously described. ${ }^{1)}$

Immunoblotting. Skeletal muscle laminin $\mathrm{M}$ was extracted by EDTA-containing buffers as previously described. ${ }^{1)}$ Samples, each containing the same amount of myosin (pre-quantitated by densitometry) were transferred to a nitrocellulose sheet and allowed to react with anti-laminin $\mathrm{M}$ antibodies for overnight at $4^{\circ} \mathrm{C}$. The reaction was visualized by the $\mathrm{ABC}$ kit (Vector Lab. U.S.A.).

Electron microscopy procedures. Glutaraldehyde fixation was used to obtain optimal preservation of fine structure as described previously with or without lantanum nitrate treatment. $^{14)}$

$R N A$ analysis for laminin $M$. Total RNA was extracted from the frozen muscle tissues by acid guanidium thiocyanate-phenol-chloroform method, and poly $\mathrm{A}^{+} \mathrm{RNA}$ was extracted for Northern blot test using the QuickPrep mRNA purification kit (Pharmacia). RT-PCR analysis was accomplished as described by Engvall et al. (1992). ${ }^{19)}$

Results. In the homozygous $d y / d y$ mice, no detectable immunostaining for laminin $\mathrm{M}$ was found in skeletal (Fig. 1) and cardiac (not shown) muscles, but was present in the heterozygous $D y / d y$ and the control $D y / D y$ mice. However, all other dystrophic animals, including $m d x$ mice, BIO 14.6 hamsters, and line 413 chickens had positive immunostaining for laminin $\mathrm{M}$ at the basal lamina and showed a sharp delineation around each muscle fiber (Fig. 1). Endoneurial basement membrane of intramuscular nerves and spinal nerve roots were also stained with anti-laminin $\mathrm{M}$ antibodies in all animals except the $d y / d y$ mice 


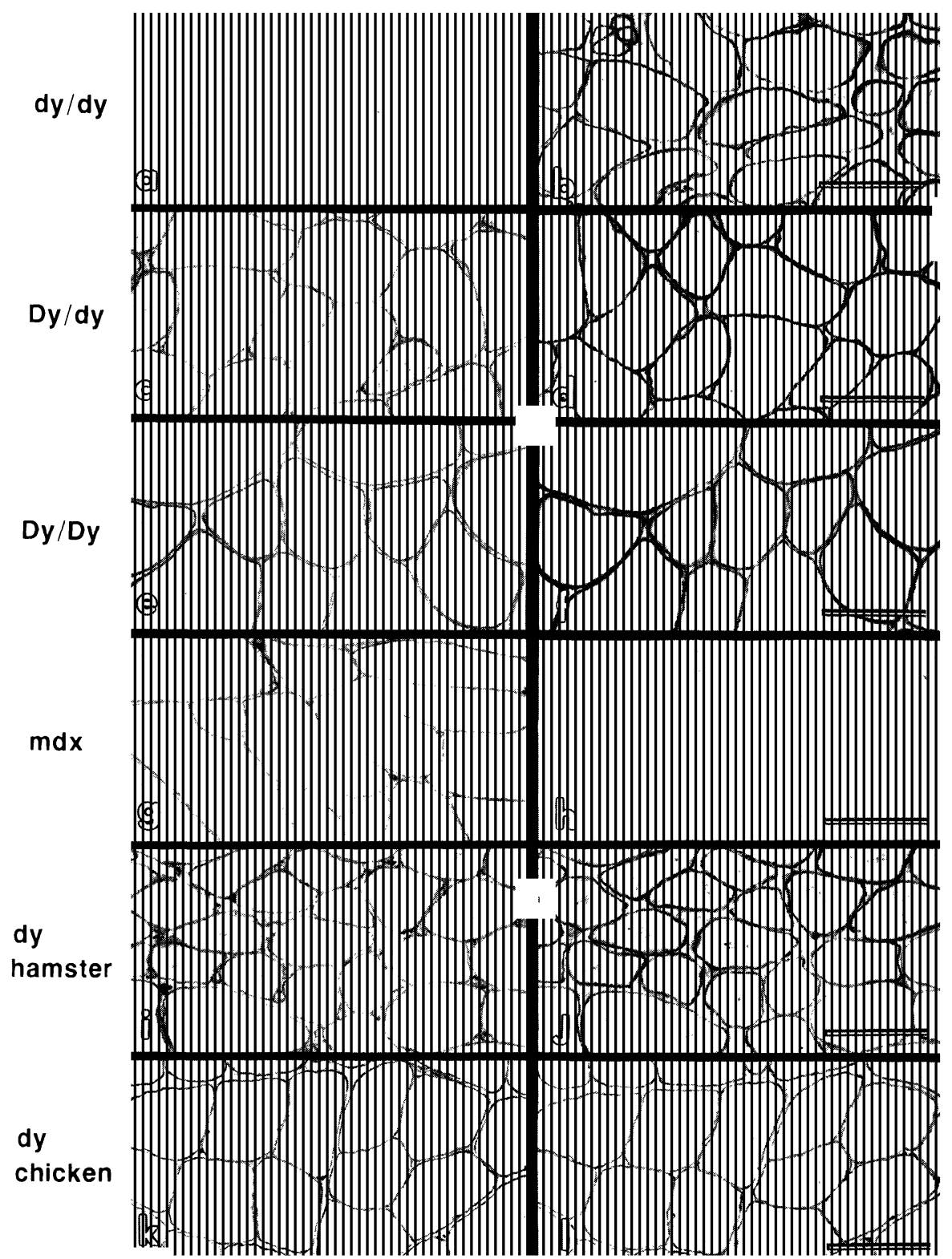

Fig. 1. Consecutive frozen sections of biopsied skeletal muscles from homozygous dystrophic $d y / d y$ (a, b), heterozygous non-affected $D y / d y$ (c, d), control $D y / D y(\mathrm{e}, \mathrm{f})$, and $m d x(\mathrm{~g}, \mathrm{~h})$ mice, and dystrophic BIO 14.6 hamster $(i, j)$ and line 413 chicken $(k, l)$ immunoreacted for laminin $M$ (a, c, e, g, i, k) and dystrophin (b, d, f, h, j, l). Note the defect of immunostaining for laminin $\mathrm{M}$ in the $d y / d y$ (a), and for dystrophin in the $m d x(\mathrm{~h})$ mice. In contrast, clear immunostaining for laminin $\mathrm{M}$ and dystrophin are similarly observed in the other specimens. Bar $=50$ microns 
[Vol. 69(B),

(Fig. 2).

Although laminin $\mathrm{M}$ was missing in the $d y / d y$ mice, other major components of the basal lamina, i.e., laminin B subunit, type IV collagen, and fibronectin were immunostained normally (data not shown).

On immunoblot analysis of skeletal muscle, $80 \mathrm{kd}$ band which corresponds to the C-terminal segment of laminin M was clearly shown in the control and heterozygous $D y / d y$ mice, but barely detectable or greatly reduced in the $d y / d y$ mice (Fig. 4a).

Electron microscopic study revealed a thinner and occasionally disrupted basal lamina in most of the $d y / d y$ muscle, while in the control $D y / D y$, thicker and continuous normal basal lamina was observed consistently (Fig. 3).

Northern blot analysis of muscle failed to detect laminin M mRNA in the $d y / d y$ mice, while RT-PCR analysis detected laminin M mRNA of similar size in both $d y / d y$ and its control Dy/Dy (Fig. 4b).

Discussion. Basal lamina is a specialized extracellular matrix that contains at least one of several variants of laminin (A, B1, B2, M, S, K) and type IV collagen in addition to fibronectin and heparan-sulfate proteoglycan and is thought to contribute to the regeneration after injury or to the development of myogenic cells. Several diseases that
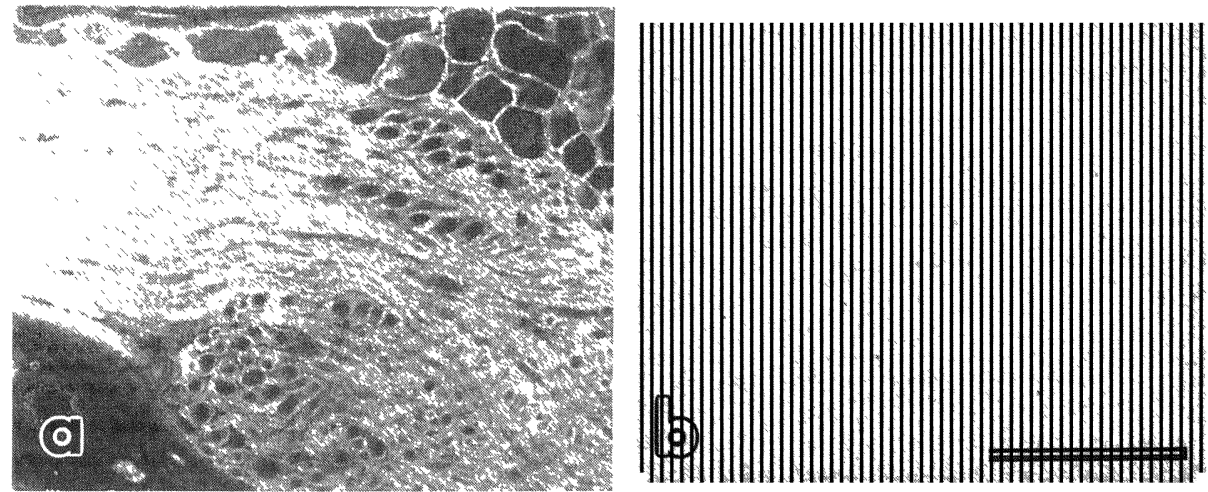

Fig. 2. Frozen sections of control $D y / D y$ (a) and nomozygous dy/dy (b) mice reacted with anti-laminin $M$ antibody. In the control, laminin $M$ immunoreaction is present in the nerve as well as the surrounding muscle, while in the $d y / d y$ no immunostaining is observed. Bar $=100$ microns

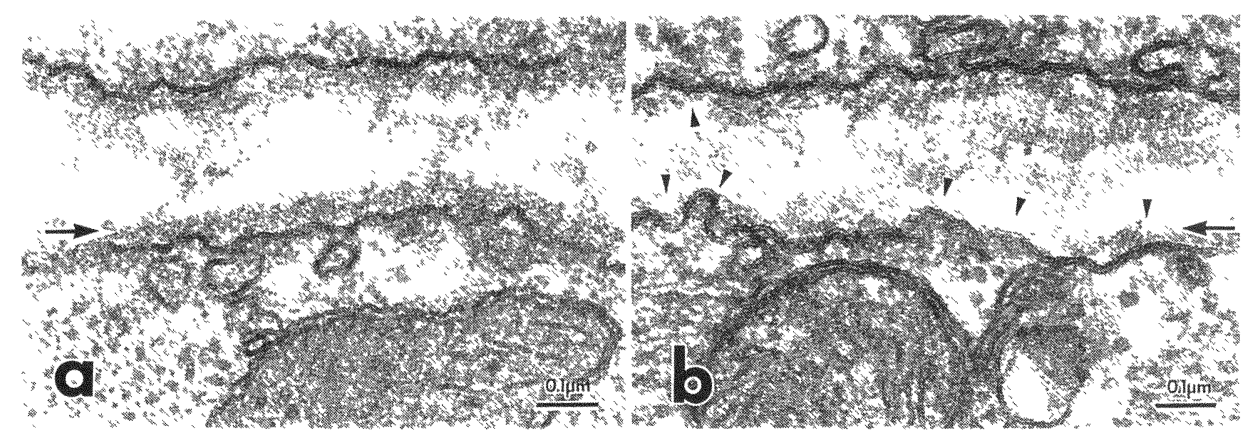

Fig. 3. Electron microscopic observation of the skeletal muscle of control $D y / D y$ (a) and homozygous $d y / d y$ (b) mice. In the control, the basal lamina at the surface of muscle fiber is intact (arrow). Contrary, thinner and disrupted (arrowhead) basal lamina (arrow) is seen in the $d y / d y$, while the underlying plasma membrane is preserved. $\mathrm{Bar}=0.1$ micron 


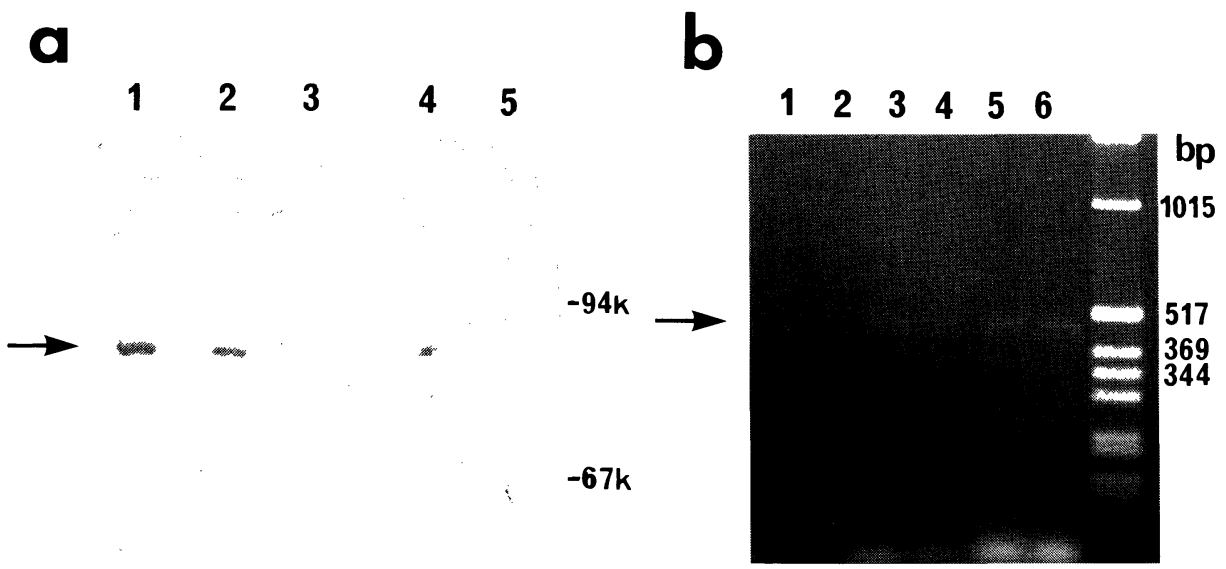

Fig. 4. (a) Immunoblot analysis of laminin $M$ using the C-terminal region (the second repeat of the $g$ domain) specific antibody. An immunoreactive component of a molecular weight of $80 \mathrm{kd} g$ domain (arrow) of laminin $\mathrm{M}$ is barely detectable in the muscle extract of 3 month-old $d y / d y$ mouse (lane 3), but are clearly observed in the age matched $D y / D y$ (lane 1) and $D y / d y$ (lane 2) mice, as well as in the control (lane 4) and the dystrophic hamsters (lane 5). (b) RT-PCR analysis of the frozen muscle tissue of a 2-week-old $d y / d y$ mouse and the control. PCR amplification are performed with 19 (lanes 1, 2), 21 (lanes 3, 4), and 23 (lanes 5, 6) cycles. Laminin M mRNA is detectable at higher PCR cycles in both the $d y / d y$ (lanes 1, 3, 5) and $D y / D y$ (lanes 2, 4, 6) mice as an expected 468bp band (arrow).

can be caused by abnormality of extracellular matrix of collagen (I, II, IV, VII), and laminin $\mathrm{K}$ have been known. ${ }^{20-24)}$ And we have found laminin M reduction in the FCMD skeletal muscle. ${ }^{1)}$ Laminin $\mathrm{M}$ is a major isoform of laminin heavy chains in the basal lamina in postnatal muscle and nerve. It should be specifically mentioned that our study demonstrated a defect of only laminin $M$ but not other components of the basal lamina in the skeletal and cardiac muscles and peripheral nerve of $d y / d y$ dystrophic mice. On the other hand, laminin $\mathrm{M}$ is not missing in non-affected $D y / d y$, and $D y / D y$ mice. Further, dystrophic $m d x$ mice, hamsters, and chickens also express laminin $\mathbf{M}$ and other components of the basal lamina normally, indicating that changes of laminin $\mathrm{M}$ is specific to the $d y / d y$ mice.

There have been an argument that dysmyelination of nerve in the $d y / d y$ mice would be unrelated to the coexisting dystrophic changes of skeletal muscle. ${ }^{15)}$ However, since we found the defect of laminin $\mathrm{M}$ in both nerve and muscle of the $d y / d y$ mice but not in the controls and heterozygoutes, it is highly conceivable that primary abnormality of laminin M could exert a pleiotropic effects on both muscle and nerve, as seen in the $d y / d y$ mice. Laminin is supposed to interact with receptor proteins on the plasma membrane of muscle fiber such as integrins ${ }^{25)}$ and $156 \mathrm{~K} \mathrm{DAG}^{26)}$ Integrin is shown to act as an efficient signal transduction molecule through the plasma membrane. ${ }^{27)}$ In the $d y / d y$ mice, M-subunit missing laminin at the basal lamina may not be able to have a physiological contact with the underlying plasma membrane. In fact, our EM results have shown a frequent detachment and gaps of basal lamina from underlying plasma membrane in the $d y / d y$ mice but not in the control and the dystrophic $m d x$ mice.

Results of Northern blot and RT-PCR analyses of muscle suggest the presence of a small amount of mRNA in the $d y / d y$ mice. Intriguing question that should be answered is to clarify how the laminin M abnormality observed in the FCMD muscle is related to the 
dystrophic changes of muscle and the morphogenetic processes of central nervous system.

Acknowledgements. We wish to thank to Dr. Tatsuji Nomura (Director of the Central Institute for Experimental Animals, Kawasaki, Japan) who contributed a great deal to establish a system of supplying $d y$ mice in Japan, and Prof. Setsuro Ebashi, M. J. A., (National Institute for Physiological Sciences, Okazaki, Japan) for his helpful discussion and advice, and to Dr. Eva Engvall for providing the antibodies.

This research has been supported by Grants from the National Center of Neurology and Psychiatry of the Ministry of Health and Welfare, Japan, the Ministry of Education, Science and Culture, Japan (K.A., H.S.).

\section{References}

1) Hayashi, Y. K. et al.: J. Neurol. Sci., 119, 53-64 (1993).

2) Michelson, A. M. et al.: Proc. Natl. Acad. Sci. U.S.A., 41, 1079-1084 (1955).

3) Buckle, V. J. et al.: Hum. Genet., 85, 324-326 (1990).

4) Ohlendieck, K. et al.: Neuron, 7, 499-508 (1991).

5) Love, D. R. et al.: Proc. Natl. Acad. Sci. U.S.A., 88, 3243-3247 (1991).

6) Bulfield, G. et al.: ibid., 81, 1189-1192 (1984).

7) Sugita, H. et al.: Proc. Japan Acad., 64B, 37-39 (1988).

8) Arahata, K. et al.: Nature, 333, 861-863 (1988).

9) Hoffman, E. P. et al.: Neuron, 1, 411-420 (1988).

10) Homburger, F. et al.: Ann. N.Y. Acad. Sci., 317, 2-17 (1979).

11) Roberds, S. L. et al.: J. Biol. Chem., 268, 11496-11499 (1993).

12) Asmundson, V. S. et al.: J. Hered., 47, 248-252 (1956).

13) Bourne, G. H. et al.: $\quad$ Muscular Dystrophy in Man and Animals. Karger, Inc., pp. 407-456 (1963).

14) Woo, M. et al.: J. Neurol. Sci., 82, 111-122 (1987).

15) Bradley, W. G. et al.: J. Neurol. Sci., 18, 227-247 (1973).

16) Bradley, W. G. et al.: Ann. N.Y. Acad. Sci., 317, 132-142 (1979).

17) Okada, E. et al.: J. Neurol. Sci., 28, 505-520 (1976).

18) Bray, G. M. et al.: J. Neuropathol. Exp. Neurol., 34, 517-530 (1975).

19) Engvall, E. et al.: Exp. Cell. Res., 198, 115-123 (1992).

20) Spotila, L. D. et al.: Proc. Natl. Acad. Sci. U.S.A., 88, 5423-5427 (1991).

21) Vandenberg, P. et al.: ibid., 88, 7640-7644 (1991).

22) Hostikka, S. L. et al.: ibid., 87, 1606-1610 (1990).

23) Christiano, A. M. et al.: Nature Genet., 4, 62-66 (1993).

24) Verrando, P. et al.: Lab. Invest., 64, 85-92 (1991).

25) Duband, J-L. et al.: Development, 116, 585-600 (1992).

26) Ibraghimov-Beskrovnaya, O. et al.: Nature, 355, 696-702 (1992).

27) Wang, N. et al.: Science, 260, 1124-1127 (1993). 